\title{
Functional version for Furuta parametric relative operator entropy
}

\author{
Mustapha Raïssouli ${ }^{1,2}$ and Shigeru Furuichi ${ }^{3 *}$
}

\author{
"Correspondence: \\ furuichi@chs.nihon-u.ac.jp \\ ${ }^{3}$ Department of Information \\ Science, College of Humanities and \\ Sciences, Nihon University, Tokyo, \\ Japan \\ Full list of author information is \\ available at the end of the article
}

\begin{abstract}
Functional version for the so-called Furuta parametric relative operator entropy is here investigated. Some related functional inequalities are also discussed. The theoretical results obtained by our functional approach immediately imply those of operator versions in a simple, fast, and nice way.
\end{abstract}

MSC: Primary 46N10; secondary 46A20; 47A63; 47N10; 39B62; 52A41

Keywords: Operator inequalities; Functional inequalities; Operator entropies; Convex analysis

\section{Introduction}

Let $H$ be a complex Hilbert space. We denote by $\mathcal{B}(H)$ the $\mathbb{C}^{*}$-algebra of bounded linear operators acting on $H$ and by $\mathcal{B}^{+*}(H)$ the open cone of all (self-adjoint) positive invertible operators in $\mathcal{B}(H)$. Let $A, B \in \mathcal{B}^{+*}(H)$ and $p \in[0,1]$ be a real number. The expressions

$$
\begin{aligned}
& A \nabla_{p} B=(1-p) A+p B, \\
& A !_{p} B=\left((1-p) A^{-1}+p B^{-1}\right)^{-1}, \\
& A \sharp_{p} B=A^{1 / 2}\left(A^{-1 / 2} B A^{-1 / 2}\right)^{p} A^{1 / 2}
\end{aligned}
$$

are known in the literature as the weighted arithmetic mean, weighted harmonic mean, and weighted geometric mean of $A$ and $B$, respectively. If $p=1 / 2$ they are simply denoted by $A \nabla B, A ! B$, and $A \sharp B$, respectively. The previous operator means satisfy the following relationships:

$$
A \nabla_{p} B=B \nabla_{1-p} A, \quad A !_{p} B=B !_{1-p} A, \quad A \sharp_{p} B=B \sharp_{1-p} A .
$$

It is well known that the double inequality

$$
A !_{p} B \leq A \sharp_{p} B \leq A \nabla_{p} B
$$

holds for any $A, B \in \mathcal{B}^{+*}(H)$ and $p \in[0,1]$. Here, the notation $T \leq S$ means that $T, S \in \mathcal{B}(H)$ are self-adjoint and $S-T$ is positive semi-definite.

(c) The Author(s) 2018. This article is distributed under the terms of the Creative Commons Attribution 4.0 International License (http://creativecommons.org/licenses/by/4.0/), which permits unrestricted use, distribution, and reproduction in any medium, provided you give appropriate credit to the original author(s) and the source, provide a link to the Creative Commons license, and indicate if changes were made. 
Otherwise, the relative operator entropy $S(A \mid B)$ and the Tsallis relative operator entropy $T_{p}(A \mid B)$ are, respectively, defined by (see $\left.[2,3,6]\right)$

$$
S(A \mid B)=A^{1 / 2} \log \left(A^{-1 / 2} B A^{-1 / 2}\right) A^{1 / 2}, \quad T_{p}(A \mid B)=\frac{A \sharp_{p} B-A}{p}, \quad p \neq 0 .
$$

The following double inequality is known in the literature:

$$
A-A B^{-1} A \leq S(A \mid B) \leq B-A \text {. }
$$

In [5], Furuta introduced a parametric extension of $S(A \mid B)$ as follows:

$$
S_{p}(A \mid B)=A^{1 / 2}\left(A^{-1 / 2} B A^{-1 / 2}\right)^{p} \log \left(A^{-1 / 2} B A^{-1 / 2}\right) A^{1 / 2} .
$$

In fact, $S_{p}(A \mid B)$ was introduced in [5] for any real number $p$, but here we restrict ourselves to the case $p \in[0,1]$.

As pointed out in [5], it is not hard to see that

$$
S_{0}(A \mid B)=S(A \mid B), \quad S_{1}(A \mid B)=-S(B \mid A) \quad \text { and } \quad S_{p}(A \mid B)=-S_{1-p}(B \mid A) .
$$

The fundamental goal of this paper is to give an extension of $S_{p}(A \mid B)$ when the operator variables $A$ and $B$ are (convex) functionals. Some functional relationships and inequalities are provided as well. The related operator versions are deduced in a fast and nice way.

\section{Functional extensions}

The previous operator concepts have been extended from the case that the variables are positive operators to the case that the variables are convex functionals, see [9].

Let $\tilde{\mathbb{R}}^{H}$ be the extended space of all functionals defined from $H$ into $\mathbb{R} \cup\{+\infty\}$. Let $f, g \in \tilde{\mathbb{R}}^{H}$ be two given functionals (convex or not) and $p \in(0,1)$. The expressions

$$
\begin{aligned}
& \mathcal{A}_{p}(f, g)=(1-p) f+p g, \\
& \mathcal{H}_{p}(f, g)=\left((1-p) f^{*}+p g^{*}\right)^{*}, \\
& \mathcal{G}_{p}(f, g)=\frac{\sin (p \pi)}{\pi} \int_{0}^{1} \frac{t^{p-1}}{(1-t)^{p}} \mathcal{H}_{t}(f, g) d t
\end{aligned}
$$

are called, by analogy, the weighted functional arithmetic mean, the weighted harmonic mean, and the weighted geometric mean of $f$ and $g$, respectively. Here, the notation $f^{*}$ refers to the Fenchel conjugate of $f$ defined by

$$
\forall x^{*} \in H \quad f^{*}\left(x^{*}\right)=\sup _{x \in H}\left\{\Re e\left\langle x^{*}, x\right\rangle-f(x)\right\}
$$

For $p=1 / 2$, we will denote the previous functional means by $\mathcal{A}(f, g), \mathcal{H}(f, g)$ and $\mathcal{G}(f, g)$, respectively. We extend these means on the whole interval $[0,1]$ by setting:

$$
\mathcal{A}_{0}(f, g)=\mathcal{H}_{0}(f, g)=\mathcal{G}_{0}(f, g)=f, \quad \mathcal{A}_{1}(f, g)=\mathcal{H}_{1}(f, g)=\mathcal{G}_{1}(f, g)=g .
$$


We mention that here we adopt the conventions $0 .(+\infty)=+\infty$ and $(+\infty)-(+\infty)=+\infty$, as usual in convex analysis $[1,8]$. With this, relations $(2.3)$ are not immediate from their related functional means (2.1) since the involved functionals $f$ and/or $g$ can take the value $+\infty$.

For the same reason, analogous relationships of (1.1) for the previous functional means are also valid, i.e.,

$$
\mathcal{A}_{p}(f, g)=\mathcal{A}_{1-p}(g, f), \quad \mathcal{H}_{p}(f, g)=\mathcal{H}_{1-p}(g, f), \quad \mathcal{G}_{p}(f, g)=\mathcal{G}_{1-p}(g, f) .
$$

In fact, the first two relations are immediate from their definitions, and for the third one, there is a detailed proof in [12]. Also, the analog of (1.2), i.e.,

$$
\mathcal{H}_{p}(f, g) \leq \mathcal{G}_{p}(f, g) \leq \mathcal{A}_{p}(f, g)
$$

holds for any $f, g \in \tilde{\mathbb{R}}^{H}$ and $p \in[0,1]$. Here the notation $f \leq g$ refers to the point-wise order between $f \in \tilde{\mathbb{R}}^{H}$ and $g \in \tilde{\mathbb{R}}^{H}$ defined by: $f \leq g$ if and only if $g(x)-f(x) \geq 0$ for all $x \in H$, with the convention $+\infty-(+\infty)=+\infty$ as already pointed before. The double inequality (2.4) implies that the three involved functional means are with finite values whenever $f$ and $g$ are so.

In the earlier papers [9] and [10] we extended $S(A \mid B)$ and $T_{p}(A \mid B)$ from operators to (convex) functionals, respectively, as follows:

$$
\begin{aligned}
& \mathcal{S}(f \mid g)=\int_{0}^{1} \frac{\mathcal{H}_{t}(f, g)-f}{t} d t, \\
& \mathcal{T}_{p}(f \mid g)=\frac{\mathcal{G}_{p}(f, g)-f}{p}, \quad p \neq 0 .
\end{aligned}
$$

The previous functional concepts were constructed as extensions of their related operator versions in the following sense: if $\mathcal{O}(A, B)$ is one of the previous operator concepts, its functional extension $\mathcal{F}(f, g)$ is such that

$$
\mathcal{F}\left(f_{A}, f_{B}\right)=f_{\mathcal{O}(A, B)}
$$

where the notation $f_{T}$, for any $T \in \mathcal{B}(H)$, refers to the quadratic function generated by the operator $T$, i.e., $f_{T}(x)=(1 / 2)\langle T x, x\rangle$ for all $x \in H$.

\section{Needed tools}

Let $f \in \tilde{\mathbb{R}}^{H}$. We denote by $\operatorname{dom} f:=\{x \in H: f(x)<+\infty\}$ the so-called effective domain of $f$. The notation $\operatorname{int}(\operatorname{dom} f)$ refers to the topological interior of $\operatorname{dom} f$ in $H$. The Fenchel conjugate $f^{*}$ of $f$ defined by (2.2) satisfies

$$
f^{*}\left(x^{*}\right):=\sup _{x \in \operatorname{dom} f}\left\{\Re e\left|x^{*}, x\right\rangle-f(x)\right\}
$$

for any $x^{*} \in H$. As supremum of a family of affine (so convex) functions, $f^{*}$ is always convex even if $f$ is not. The conjugate map $f \longmapsto f^{*}$ is point-wise decreasing and convex. That is, 
$f \leq g$ implies $g^{*} \leq f^{*}$, and the inequality

$$
((1-p) f+p g)^{*} \leq(1-p) f^{*}+p g^{*}
$$

holds for any $f, g \in \tilde{\mathbb{R}}^{H}$ and $p \in[0,1]$.

The sub-differential of $f$ at $x \in \operatorname{dom} f$ is the set $\partial f(x)$ defined by

$$
\partial f(x)=\left\{x^{*} \in H ; \forall z \in H, \quad f(z) \geq f(x)+\Re e\left(x^{*}, z-x\right)\right\} .
$$

As it is well known, $\partial f(x)$ is a (possibly empty) convex and closed set. If $x \in \operatorname{int}(\operatorname{dom} f)$, then $\partial f(x) \neq \emptyset$. In the case where $\partial f(x) \neq \emptyset$, we have the equivalence:

$$
x^{*} \in \partial f(x) \Longleftrightarrow f(x)+f^{*}\left(x^{*}\right)=\Re e\left(x^{*}, x\right) .
$$

As usual we denote by $\Gamma_{0}(H)$ the cone of all functionals $f \in \tilde{\mathbb{R}}^{H}$ that are convex, lower semi-continuous, and proper (i.e., not identically equal to $+\infty$ ). It is well known that $f^{* *}:=$ $\left(f^{*}\right)^{*} \leq f$ for any $f \in \tilde{\mathbb{R}}^{H}$ and $f \in \Gamma_{0}(H)$ if and only if $f=f^{* *}:=\left(f^{*}\right)^{*}$. Moreover, $x^{*} \in \partial f(x)$ always implies $x \in \partial f^{*}\left(x^{*}\right)$, with reversed implication provided that $f \in \Gamma_{0}(H)$.

The function $f$ is called Gâteaux-differentiable (in short G-differentiable) at $x$ if the directional derivative

$$
f^{\prime}(x, d)=\lim _{t \downarrow 0} \frac{f(x+t d)-f(x)}{t}
$$

of $f$ at $x$ exists in every direction $d \in H$ and the map $d \longmapsto f^{\prime}(x, d)$ is linear and continuous. In this case we write $f^{\prime}(x, d)=\nabla f(x) \cdot d$ and $\nabla f(x)$ is called the G-derivative of $f$ at $x$. It is well known that if $f$ is convex and G-differentiable at $x$, then $\partial f(x)=\{\nabla f(x)\}$.

For the sake of clearness and simplicity for the reader, we state the following example illustrating the previous concepts.

Example 3.1 Let $A \in \mathcal{B}(H)$ and let $f_{A}$ be the quadratic function associated to $A$, i.e., $f_{A}(x)=$ $(1 / 2)\langle A x, x\rangle$ for all $x \in H$.

(i) Assume that $A \in \mathcal{B}^{+*}(H)$. Then $f_{A}$ is convex and G-differentiable on $H$, and so

$$
\forall x \in H \quad \partial f_{A}(x)=\left\{\nabla f_{A}(x)\right\}=\{A x\}
$$

The coefficient $1 / 2$ appearing in $f_{A}$ enjoys a symmetry role in the aim to have

$$
\left(f_{A}\right)^{*}\left(x^{*}\right)=(1 / 2)\left\langle A^{-1} x^{*}, x^{*}\right\rangle \text { for all } x^{*} \in H \text {, or in short }\left(f_{A}\right)^{*}=f_{A^{-1}} .
$$

(ii) For any $A, B \in \mathcal{B}(H)$, it is easy to check that $f_{A} \pm f_{B}=f_{A \pm B}$ and $f_{A}(B x)=f_{B A B}(x)$ for any $x \in H$.

The following result, which will be needed later, has been proved in [11].

Theorem 3.2 Let $f \in \Gamma_{\circ}(H)$ be such that $\operatorname{int}(\operatorname{dom} f)$ is nonempty. Then 
(i) The inequality

$$
\sup _{x^{*} \in \partial f(x)}\left(f^{*}-g^{*}\right)\left(x^{*}\right) \leq \mathcal{S}(f / g)(x) \leq(g-f)(x)
$$

holds true for all $x \in \operatorname{int}(\operatorname{dom} f)$.

(ii) Iff is moreover G-differentiable at $x$, then we have

$$
f^{*}(\nabla f(x))-g^{*}(\nabla f(x)) \leq \mathcal{S}(f / g)(x) \leq(g-f)(x)
$$

As explained in [11], (3.1), as well as (3.2), is a functional extension of (1.3) from positive operators to convex functionals.

For the sake of simplicity for the reader, we need to introduce an auxiliary notation. For $f, g \in \tilde{\mathbb{R}}^{H}$ and $p \in[0,1]$, we set

$$
\mathcal{T}_{p}^{*}(f \mid g)=\frac{\left(\mathcal{G}_{p}(f, g)\right)^{*}-f^{*}}{p}, \quad p \neq 0 .
$$

We have the following result summarizing the elementary properties of $\mathcal{T}_{p}^{*}(f \mid g)$.

\section{Proposition 3.3 The following assertions hold:}

(i) For any $p \in[0,1)$, one has

$$
\mathcal{T}_{1-p}^{*}(g \mid f)=\frac{\left(\mathcal{G}_{p}(f, g)\right)^{*}-g^{*}}{1-p} .
$$

(ii) For all $p \in(0,1]$, the left-hand side of the inequality

$$
\frac{\left(\mathcal{A}_{p}(f, g)\right)^{*}\left(x^{*}\right)-f^{*}\left(x^{*}\right)}{p} \leq \mathcal{T}_{p}^{*}(f \mid g)\left(x^{*}\right) \leq g^{*}\left(x^{*}\right)-f^{*}\left(x^{*}\right)
$$

holds for any $x^{*} \in H$, while the right-hand side holds for $x^{*}$ such that $g^{*}\left(x^{*}\right)=+\infty$ or $x^{*} \in \operatorname{dom} f^{*}$.

Proof (i) Follows from (3.3) with the relation $\mathcal{G}_{p}(f, g)=\mathcal{G}_{1-p}(g, f)$.

(ii) From (2.4) we obtain by taking the conjugate side by side

$$
\left(\mathcal{A}_{p}(f, g)\right)^{*} \leq\left(\mathcal{G}_{p}(f, g)\right)^{*} \leq\left(\mathcal{H}_{p}(f, g)\right)^{*}
$$

Remarking that

$$
\left(\mathcal{H}_{p}(f, g)\right)^{*} \leq(1-p) f^{*}+p g^{*}
$$

we then deduce the desired result.

Proposition 3.4 For any $A, B \in \mathcal{B}^{+*}(H)$ and $p \in(0,1]$, there holds

$$
\mathcal{T}_{p}^{*}\left(f_{A} \mid f_{B}\right)=f_{T_{p}\left(A^{-1} \mid B^{-1}\right)}
$$


Proof First, if for fixed $p \in[0,1]$ we take $\mathcal{F}=\mathcal{G}_{p}$ and $\mathcal{O}(A, B)=A \sharp_{p} B$ in (2.5), then we have

$$
\mathcal{G}_{p}\left(f_{A}, f_{B}\right)=f_{A \sharp p} \text {. }
$$

Now, by (3.3) we have

$$
\mathcal{T}_{p}^{*}\left(f_{A} \mid f_{B}\right)=\frac{\left(\mathcal{G}_{p}\left(f_{A}, f_{B}\right)\right)^{*}-f_{A}^{*}}{p}=\frac{f_{A \sharp p}^{*}-f_{A}^{*}}{p}=\frac{f_{\left(A_{\sharp} B\right)^{-1}}-f_{A^{-1}}}{p}=\frac{f_{A^{-1} \sharp_{p} B^{-1}}-f_{A^{-1}}}{p} .
$$

This, with the fact that $\alpha f_{T}=f_{\alpha T}$ and $f_{T}-f_{S}=f_{T-S}$ for any $\alpha \in \mathbb{R}$ and $T, S \in \mathcal{B}(H)$, immediately yields the desired result.

\section{Functional version of $S_{p}(A \mid B)$}

As already pointed out before, our aim here is to give an analog of $S_{p}(A \mid B)$ when the operator arguments $A$ and $B$ are (convex) functionals $f$ and $g$, respectively. Such an analog seems to be hard to define from (1.4) since (1.4) involves the product of operators whose analogs for functionals are not known yet. For this, we need to state the following result.

Theorem 4.1 The equalities

$$
S_{p}(A \mid B)=-\frac{S\left(A \sharp_{p} B \mid A\right)}{p}=\frac{S\left(A \sharp_{p} B \mid B\right)}{1-p}
$$

hold for any $A, B \in \mathcal{B}^{+*}(H)$ and $p \in(0,1)$.

Proof Indeed, we have the property

$$
T^{*} S(A \mid B) T=S\left(T^{*} A T \mid T^{*} B T\right)
$$

for any $A, B \in \mathcal{B}^{*}(H)$ and any invertible operator $T \in \mathcal{B}(H)$ by using Kubo-Ando theory [7] and the integral form

$$
S(A \mid B)=\int_{0}^{1} \frac{A !_{t} B-A}{t} d t
$$

We thus have the first equality as

$$
\begin{aligned}
S\left(A \sharp_{p} B \mid A\right) & =A^{1 / 2} S\left(I \sharp_{p} A^{-1 / 2} B A^{-1 / 2} \mid I\right) A^{1 / 2} \\
& =A^{1 / 2} S\left(\left(A^{-1 / 2} B A^{-1 / 2}\right)^{p} \mid I\right) A^{1 / 2} \\
& =-A^{1 / 2}\left(A^{-1 / 2} B A^{-1 / 2}\right)^{p} \log \left(A^{-1 / 2} B A^{-1 / 2}\right)^{p} A^{1 / 2} \\
& =-p S_{p}(A \mid B),
\end{aligned}
$$

since $S(A \mid I)=-A \log A$ for any $A \in \mathcal{B}^{+*}(H)$.

The second equality can be proved in a similar manner.

Now, to give a functional version of $S_{p}(A \mid B)$, we use (4.1) which is more appropriate for our aim since (4.1) involves only operator concepts (relative operator entropy and operator geometric mean) whose functional extensions are already done. Taking into account 
a symmetric character between $p$ and $1-p$ in our desired definition, we then put the following.

Definition 4.2 Let $f, g \in \tilde{\mathbb{R}}^{H}$ and $p \in[0,1]$. We set

$$
\mathcal{S}_{p}(f \mid g)=\frac{\mathcal{S}\left(\left(\mathcal{G}_{p}(f, g)\right) \mid g\right)}{2(1-p)}-\frac{\mathcal{S}\left(\left(\mathcal{G}_{p}(f, g)\right) \mid f\right)}{2 p},
$$

with

$$
\mathcal{S}_{0}(f \mid g)=\mathcal{S}(f \mid g) \quad \text { and } \quad \mathcal{S}_{1}(f \mid g)=-\mathcal{S}(g \mid f)
$$

As a first result we state the following.

Proposition 4.3 Let $f, g \in \tilde{\mathbb{R}}^{H}$. Then we have

$$
\mathcal{S}_{1 / 2}(f \mid g)=\mathcal{S}((\mathcal{G}(f, g)) \mid g)-\mathcal{S}((\mathcal{G}(f, g)) \mid f) .
$$

Further, if $\operatorname{dom} f=\operatorname{dom} g=H$, then the equality

$$
\mathcal{S}_{p}(f \mid g)=-\mathcal{S}_{1-p}(g \mid f)
$$

holds for any $p \in(0,1)$.

Proof Equality (4.3) is immediate from (4.2). However, we mention that (4.4) is not immediate from (4.2) since our involved functionals could take the value $+\infty$. Indeed, we pay attention to the fact that, if $\phi, \psi \in \tilde{\mathbb{R}}^{H}$, the equality $\phi-\psi=-(\psi-\phi)$ is not always true unless $\operatorname{dom} \phi \cup \operatorname{dom} \psi=H$. For this reason, we have assumed in our statement that $\operatorname{dom} f=\operatorname{dom} g=H$ in the aim to guarantee that $\mathcal{H}_{t}\left(\mathcal{G}_{p}(f, g), g\right)$ or $\mathcal{H}_{t}\left(\mathcal{G}_{p}(f, g), f\right)$ is with finite values. With this, (4.4) can be deduced from (4.2) when we refer to the relationship $\mathcal{G}_{p}(\phi, \psi)=\mathcal{G}_{1-p}(\psi, \phi)$ valid for any $\phi, \psi \in \tilde{\mathbb{R}}^{H}$ and $p \in[0,1]$.

A connection between the functional parametric entropy $\mathcal{S}_{p}(f \mid g)$ and the operator parametric entropy $S_{p}(A \mid B)$ is expressed by the following result.

Proposition 4.4 Let $A, B \in \mathcal{B}^{+*}(H)$ and $p \in[0,1]$. Then we have

$$
\mathcal{S}_{p}\left(f_{A} \mid f_{B}\right)=f_{S_{p}(A \mid B)}
$$

Proof By (4.2), with (2.5) and (4.1), we have

$$
\mathcal{S}_{p}\left(f_{A} \mid f_{B}\right)=\frac{\mathcal{S}\left(f_{A \sharp_{p} B} \mid f_{B}\right)}{2(1-p)}-\frac{\mathcal{S}\left(f_{A \sharp_{p} B} \mid f_{A}\right)}{2 p}=\frac{f_{S\left(A \sharp_{p} B \mid B\right)}}{2(1-p)}-\frac{f_{S\left(A \sharp_{p} B \mid A\right)}}{2 p} .
$$

This, with similar arguments as in the proof of Proposition 3.4, implies the desired result.

Relationship (4.5) justifies that $\mathcal{S}_{p}(f \mid g)$ is a reasonable extension of $S_{p}(A \mid B)$, from operators to functionals, in the sense of (2.5). 
For the sake of simplicity, we use in the next theorem and in its proof the following notations:

$$
\mathcal{G}_{p}:=\mathcal{G}_{p}(f, g), \quad \mathcal{G}_{p}^{*}:=\left(\mathcal{G}_{p}(f, g)\right)^{*}, \quad \nabla \mathcal{G}_{p}:=\nabla\left(\mathcal{G}_{p}(f, g)\right), \quad \partial \mathcal{G}_{p}:=\partial\left(\mathcal{G}_{p}(f, g)\right)
$$

We now are in a position to state the following main result.

Theorem 4.5 Let $f, g \in \tilde{\mathbb{R}}^{H}$ be such that $\operatorname{int}\left(\operatorname{dom} \mathcal{G}_{p}(f, g)\right) \neq \emptyset$. Then the following double inequality

$$
\begin{aligned}
& \frac{1}{2}\left(\sup _{x^{*} \in \partial \mathcal{G}_{p}(x)} \mathcal{T}_{1-p}^{*}(g \mid f)\left(x^{*}\right)+\mathcal{T}_{p}(f \mid g)(x)\right) \\
& \quad \leq \mathcal{S}_{p}(f \mid g)(x) \\
& \quad \leq \frac{1}{2}\left(-\mathcal{T}_{1-p}(g \mid f)(x)-\sup _{x^{*} \in \partial \mathcal{G}_{p}(x)} \mathcal{T}_{p}^{*}(f \mid g)\left(x^{*}\right)\right)
\end{aligned}
$$

holds for any $x \in \operatorname{int}\left(\operatorname{dom} \mathcal{G}_{p}(f, g)\right)$ and $p \in(0,1)$.

Proof Since $\operatorname{int}\left(\operatorname{dom} \mathcal{G}_{p}(f, g)\right) \neq \emptyset$, then $\partial \mathcal{G}_{p}(x) \neq \emptyset$ for any $x \in \operatorname{int}\left(\operatorname{dom} \mathcal{G}_{p}(f, g)\right)$.

Now, according to Theorem 3.2, we have, for $x \in \operatorname{int}\left(\operatorname{dom} \mathcal{G}_{p}(f, g)\right)$,

$$
\sup _{x^{*} \in \partial \mathcal{G}_{p}(x)}\left(\mathcal{G}_{p}^{*}-f^{*}\right)\left(x^{*}\right) \leq \mathcal{S}\left(\mathcal{G}_{p} \mid f\right)(x) \leq\left(f-\mathcal{G}_{p}\right)(x)
$$

and

$$
\sup _{x^{*} \in \partial \mathcal{G}_{p}(x)}\left(\mathcal{G}_{p}^{*}-g^{*}\right)\left(x^{*}\right) \leq \mathcal{S}\left(\mathcal{G}_{p} \mid g\right)(x) \leq\left(g-\mathcal{G}_{p}\right)(x) .
$$

Multiplying (4.7) by $-1 / p$ and (4.8) by $1 /(1-p)$ and then summing side by side, we obtain the desired inequalities after simple manipulations with the help of Proposition 3.3. The details are simple and therefore omitted.

Remark 4.6 It is worth mentioning that the condition $\operatorname{int}\left(\operatorname{dom} \mathcal{G}_{p}(f, g)\right) \neq \emptyset$ is satisfied if $\operatorname{int}(\operatorname{dom} f \cap \operatorname{dom} g) \neq \emptyset$ since $\operatorname{dom} f \cap \operatorname{dom} g \subset \operatorname{dom} \mathcal{G}_{p}(f, g)$.

Corollary 4.7 Let $f, g \in \tilde{\mathbb{R}}^{H}$ be such that $\mathcal{G}_{p}(f, g)$ is G-differentiable at $x \in H$. Then the inequalities (in the point-wise order sense)

$$
\begin{aligned}
& \frac{1}{2}\left(\mathcal{T}_{1-p}^{*}(g \mid f)\left(\nabla \mathcal{G}_{p}(f, g)\right)+\mathcal{T}_{p}(f \mid g)\right) \\
& \quad \leq \mathcal{S}_{p}(f \mid g) \\
& \quad \leq \frac{1}{2}\left(-\mathcal{T}_{1-p}(g \mid f)-\mathcal{T}_{p}^{*}(f \mid g)\left(\nabla \mathcal{G}_{p}(f, g)\right)\right)
\end{aligned}
$$

hold for any $p \in(0,1)$. 
Proof Since $\mathcal{G}_{p}(f, g)$ is G-differentiable at $x$, then $\partial \mathcal{G}_{p}(f, g)(x)=\left\{\nabla \mathcal{G}_{p}(f, g)(x)\right\}$. Substituting this in (4.6) and using the definition of the point-wise order, we immediately obtain the desired inequalities.

The operator version of the above theorem (and corollary) reads as follows.

Corollary 4.8 Let $A, B \in \mathcal{B}^{+*}(H)$ and $p \in(0,1)$. Then we have

$$
\begin{aligned}
& \frac{1}{2}\left(\left(A \sharp_{p} B\right) T_{1-p}\left(B^{-1} \mid A^{-1}\right)\left(A \sharp_{p} B\right)+T_{p}(A \mid B)\right) \\
& \quad \leq S_{p}(A \mid B) \\
& \quad \leq \frac{1}{2}\left(-T_{1-p}(B \mid A)-\left(A \sharp_{p} B\right) T_{p}\left(A^{-1} \mid B^{-1}\right)\left(A \sharp_{p} B\right)\right) .
\end{aligned}
$$

Proof Combining Corollary 4.7, Proposition 3.4, and Example 3.1,(ii), we obtain the desired operator inequalities after simple manipulations. The details are simple and therefore omitted.

Corollary 4.8 gives the relation between Furuta parametric relative operator entropy and Tsallis relative operator entropy in a more general setting than the result in $[4$, Theorem 2.3].

\section{Acknowledgements}

The authors thank anonymous referees for giving valuable comments and suggestions to improve our manuscript.

Funding

The author (S.F.) was partially supported by JSPS KAKENHI Grant Number 16 K05257.

Competing interests

The authors declare that they have no competing interests.

Authors' contributions

The work presented here was carried out in collaboration between all authors. All authors have contributed to, checked, read and approved the manuscript.

\section{Author details}

${ }^{1}$ Department of Mathematics, Science Faculty, Taibah University, Al Madinah Al Munawwarah, Saudi Arabia. ${ }^{2}$ Department of Mathematics, Science Faculty, Moulay Ismail University, Meknes, Morocco. ${ }^{3}$ Department of Information Science,

College of Humanities and Sciences, Nihon University, Tokyo, Japan.

\section{Publisher's Note}

Springer Nature remains neutral with regard to jurisdictional claims in published maps and institutional affiliations.

Received: 3 April 2018 Accepted: 6 August 2018 Published online: 15 August 2018

\section{References}

1. Ekeland, I., Temam, R.: Convex Analysis and Variational Problems. SIAM, Philadelphia (1999)

2. Fujii, J.I., Kamei, E.: Relative operator entropy in noncommutative information theory. Math. Jpn. 34, 341-348 (1989)

3. Furuichi, S.: Inequalities for Tsallis relative entropy and generalized skew information. Linear Multilinear Algebra 59(10), 1143-1158 (2011)

4. Furuichi, S., Minculete, N.: Inequalities for relative operator entropies and operator means. Acta Math. Vietnam. (2018). https://doi.org/10.1007/s40306-018-0250-7

5. Furuta, T.: Parameteric extensions of Shannon inequality and its reverse one in Hilbert space operators. Linear Algebra Appl. 381, 219-235 (2004)

6. Isa, H., Ito, M., Kamei, E., Tohyama, H., Watanabe, M.: Shannon type inequalities of a relative operator entropy including Tsallis and Rényi ones. Ann. Funct. Anal. 6(4), 289-300 (2015)

7. Kubo, F., Ando, T.: Means of positive linear operators. Math. Ann. 246, 205-224 (1980)

8. Laurent, P.J.: Approximation et Optimisation. Hermann, Paris (1972)

9. Raïssouli, M.: Tsallis relative entropy for convex functionals. Int. J. Pure Appl. Math. 51(4), 555-563 (2009) 
10. Raïssouli, M.: Relative functional entropy in convex analysis. J. Appl. Anal. 17, 231-239 (2011)

11. Raïssouli, M.: Functional versions for some operator entropy inequalities. Ann. Funct. Anal. 6(2), 204-211 (2015)

12. Raïssouli, M., Bouziane, H.: Arithmetico-geometrico-harmonic functional mean in convex analysis. Ann. Sci. Math. Qué. 30(1), 79-107 (2006)

Submit your manuscript to a SpringerOpen ${ }^{\circ}$ journal and benefit from:

- Convenient online submission

$\checkmark$ Rigorous peer review

- Open access: articles freely available online

- High visibility within the field

Retaining the copyright to your article

Submit your next manuscript at $\boldsymbol{\Delta}$ springeropen.com 